\title{
Potencial das aplicações de imagens do satélite EROS em estudos geográficos
}

\author{
Marcelo Fernando Fonseca ${ }^{*}$ \\ Lindon Fonseca Matias ${ }^{* *}$
}

\section{Resumo}

O conjunto de satélites EROS (Earth Remote Observation Satellite), de origem israelense, apresenta como característica principal uma refinada resolução espacial. As imagens obtidas por este satélite ainda são pouco conhecidas e utilizadas no Brasil. O objetivo deste artigo é apresentar as características básicas das imagens adquiridas por esse satélite, abordando algumas de suas vantagens e desvantagens em comparação a outros sensores de alta resolução espacial. Com isso pretende-se demonstrar o potencial de utilização desses dados em estudos geográficos e apresentar alguns trabalhos já desenvolvidos em território nacional e internacional com o uso dessas imagens.

Palavras-chave: Satélite e imagem EROS; Alta resolução espacial; Planejamento urbano; Estudos geográficos.

Potential of the application of EROS satellite images in geographical studies

\begin{abstract}
The series of EROS Satellite (Earth Remote Observation Satellite), created in Israel, has as main characteristic an excellent spatial resolution. The images obtained by this satellite are very

* Mestrando em Geografia pela Universidade Estadual de Campinas UNICAMP (marcelo.fonseca@ige.unicamp.br)

** Professor do Departamento de Geografia do Instituto de Geociências UNICAMP (lindon@ige.unicamp.br).
\end{abstract}

Geosul, Florianópolis, v. 24, n. 47, p 131-145, jan./jun. 2009 
FONSECA, M.F. \& MATIAS, L.F. Potencial das aplicações de imagens ...

little known and used in Brazil. The article objective is to show us the basic characteristics of the images obtained, considering some advantages and disadvantages in comparison with other sensors of high resolution. So, this article intends to show us the large use of this information in geographical studies and some projects already developed in national and international territory using these images.

Keywords: EROS Satellite and Images; High spatial resolution; Urban planning; Geographical studies.

\section{Apresentação do satélite EROS}

A família de satélites EROS (Earth Remote Observation Satellite) foi lançada de uma base localizada ao leste da Sibéria portanto, com colaboração de cientistas russos - em 05 de dezembro de 2000. Projetado e desenvolvido pela empresa estatal Israel Aircraft Industries (IAI), para fins militares e civis, apresenta um sensor de alta resolução espacial (variando de 0,82 a $1,8 \mathrm{~m}$ ) e satisfatória resolução temporal.

Com ampla capacidade de imagear extensas áreas geográficas, o projeto EROS priorizou a competitividade no mercado mundial através do fornecimento de produtos similares aos já consagrados satélites americanos Ikonos e Quickbird. Concebido inicialmente como um projeto de caráter militar, suas imagens começam agora a se difundir na área civil em diversos países.

A empresa responsável pela operação do satélite - ImageSat International - disponibiliza em seu sítio (www.imagesatintl.com) alguns exemplos atuais das aplicações das imagens EROS em diferentes países, algumas das quais serão apresentadas e comentadas na seqüência deste artigo. A empresa mantém em sua base operacional o desenvolvimento de novas tecnologias espaciais, com foco nas áreas de sensores e softwares, na busca de plataformas "leves" e de reduzido custo econômico (IMAGESAT, 2007). 
FONSECA, M.F. \& MATIAS, L.F. Potencial das aplicações de imagens ...

No Brasil, uma das instituições pioneiras na utilização dessas imagens dentro da área científica é a Embrapa Monitoramento por Satélite, cuja unidade de estudos localiza-se no município de Campinas, SP.

A concepção do EROS baseou-se, essencialmente, na busca de melhores resultados em termos de resolução espacial (chegando à casa dos centímetros) e resolução temporal (buscando um recobrimento freqüente da superfície terrestre). O lançamento do conjunto de satélites EROS dividiu-se em duas categorias, denominadas de A e B. Os da família A obtêm imagens com resolução espacial de até $1,8 \mathrm{~m}$, e os da família $\mathrm{B}$ o fazem até 0,82 $\mathrm{m}$, atingindo significativo patamar frente a alguns dos principais satélites de alta resolução disponíveis atualmente - Ikonos e QuickBird -, que apresentam sensores com resolução espacial de $1,0 \mathrm{~m}$ e $0,61 \mathrm{~m}$, respectivamente (ENGESAT, 2007).

A faixa imageada compreende cenas com extensão de até $12,5 \mathrm{~km}$ para o satélite EROS A e de até $16 \mathrm{~km}$ para os da família EROS B, podendo ser utilizadas para estudos de grandes áreas, como no caso de monitoramento de recursos naturais e planejamentos ambientais e territoriais. Em relação à resolução espectral, para ambas as categorias, há variação de 0,5 até 0,9 microns, com bandas espectrais no visível e infravermelho (câmara CCD e TDI - Charge Coupled Device e Time Delay Integration).

Outra característica deste satélite se refere à baixa interferência ou ruído na captação das imagens, o que permite boa nitidez e ganhos qualitativos ao se trabalhar com efeitos como contraste e brilho em relação aos níveis de cinza da imagem. Este parâmetro está associado ao posterior processamento digital da imagem, que acaba apresentando melhores resultados na correção das distorções, degradações e ruídos introduzidos na imagem durante o processo de imageamento (ROSA, 1996).

A precisão no quesito georreferenciamento é da ordem de $100 \mathrm{~m}$, considerando uma cena de 12,5 por $12,5 \mathrm{~km}$; portanto, para estudos de precisão posicional, requerem-se ajustes através de outras técnicas complementares. O satélite EROS apresenta 
FONSECA, M.F. \& MATIAS, L.F. Potencial das aplicações de imagens ...

"flexibilidade" no imageamento, já que o mesmo pode ser orientado em até $45^{\circ}$ para qualquer direção de sua órbita, imageando áreas distintas em uma mesma passagem, além de ajustes adequados quanto às condições de iluminação. Também é possível obter pares estéreos para finalidade específica, como a extração de curvas de nível (EMBRAPA, 2007).

Pela tecnologia empregada, o satélite EROS pode adquirir, em uma única passagem, até 28 cenas compreendendo 12,5 x 12,5 $\mathrm{km}$ (EROS A) ou até 58 cenas de $16 \times 16 \mathrm{~km}$ (EROS B). Com todos os satélites operando, é possível imagear uma mesma área até duas vezes por dia, o que implica significativos ganhos temporais. Há estações de recepção das imagens na América do Norte, América do Sul, Europa, Oriente Médio, África e Austrália, e podem ser solicitados recortes com pedidos mínimos de $5 \mathrm{~km}^{2}$ (IMAGESAT, 2007). O Quadro 01 apresenta uma síntese comparativa com os principais dados referentes aos satélites citados neste artigo.

Um dos fatores que inibe uma maior divulgação dos produtos EROS pode estar no fato das imagens possuírem somente característica pancromática (níveis de cinza). $\mathrm{O}$ mercado de imagens ainda carrega tendências muito fortes com relação à priorização na aquisição de cenas coloridas, como as provenientes de aerolevantamentos ou mesmo dos satélites Ikonos e QuickBird, que realizam coleta de cenas multiespectrais e por isso permitem a fusão de bandas para obtenção de composições híbridas. Embora possa servir e atender plenamente os mesmos objetivos, as imagens pancromáticas ainda são vistas de forma diferenciada por alguns usuários, que desqualificam o produto sem avaliar corretamente suas características técnicas, incorrendo, muitas vezes, em um grave erro analítico.

O que chama a atenção é que, pelo fato de serem coletadas no formato pancromático, as imagens EROS podem apresentar redução nos custos de aquisição, em relação às imagens de satélites de resolução similar, em mais de $30 \%$, sendo este um importante fator competitivo. 
FONSECA, M.F. \& MATIAS, L.F. Potencial das aplicações de imagens ...

Quadro 01: Comparação entre os satélites de alta resolução espacial Quickbird, Ikonos II e EROS

\begin{tabular}{|c|c|c|c|c|}
\hline Sensor & $\begin{array}{c}\text { Bandas / Resolução } \\
\text { Espectral }\end{array}$ & $\begin{array}{l}\text { Resolução } \\
\text { Espacial }\end{array}$ & $\begin{array}{l}\text { Resolução } \\
\text { Temporal } \\
\end{array}$ & $\begin{array}{c}\text { Faixa } \\
\text { Imageada }\end{array}$ \\
\hline $\begin{array}{l}\text { QUICKBIRD } \\
\text { (DigitalGlobe) }\end{array}$ & $\begin{array}{c}\text { Pan } 0.45-0.90 \mu \\
\text { Azul } 0.45-0.52 \mu \\
\text { Verde } 0.52-0.60 \mu \\
\text { Verm. } 0.63-0.69 \mu\end{array}$ & $\begin{array}{c}0,61 \mathrm{~m} \\
\text { (Pancromática) } \\
2,40 \mathrm{~m} \text { (Multiespectral) }\end{array}$ & $\begin{array}{c}1 \text { a 3,5 } \\
\text { dias - } \\
\text { (variando } \\
\text { com a } \\
\text { latitude) }\end{array}$ & $\begin{array}{c}16,5 \times 16,5 \\
\mathrm{~km}\end{array}$ \\
\hline $\begin{array}{l}\text { IKONOS II } \\
\text { (Space } \\
\text { Imaging) }\end{array}$ & $\begin{array}{c}\text { Pan } 0.45-0.90 \mu \\
\text { Azul } 0.45-0.52 \mu \\
\text { Verde } 0.52-0.60 \mu \\
\text { Verm. } 0.63-0.69 \mu \\
\text { Infra verm. próx. } \\
\quad 0.76-0.90 \mu \\
\end{array}$ & $\begin{array}{c}1,0 \mathrm{~m} \\
\text { (Pancromática) } \\
4,0 \mathrm{~m} \\
\text { (Multiespectral) }\end{array}$ & $\begin{array}{l}2,9 \text { dias - } \\
\text { (variando } \\
\text { com a } \\
\text { latitude) }\end{array}$ & $13 \times 13 \mathrm{~km}$ \\
\hline $\begin{array}{l}\text { EROS } \\
\text { (ImageSat } \\
\text { International) }\end{array}$ & $\begin{array}{c}\text { Pan e Infravermelho } \\
0.50-0.90 \mu\end{array}$ & $\begin{array}{c}0,82 \mathrm{~m} \text { - EROS B } \\
\text { (Pancromática) } \\
1,80 \mathrm{~m} \text { - EROS A } \\
\text { (Pancromática) }\end{array}$ & $\begin{array}{c}\text { Diária - } \\
\text { (com todos } \\
\text { os satélites } \\
\text { operando) }\end{array}$ & $\begin{array}{l}12,5 \times 12,5 \\
\mathrm{~km} \\
(\text { EROS A) } \\
\mathrm{e} \\
16 \times 16 \mathrm{~km} \\
\text { (EROS B) }\end{array}$ \\
\hline \multicolumn{5}{|c|}{ Principais Aplicações } \\
\hline \multicolumn{5}{|c|}{$\begin{array}{l}\text { - Mapeamentos de alta precisão; } \\
\text { - Mapeamentos detalhados da malha urbana e infra-estrutura; } \\
\text { - Uso e ocupação da terra; } \\
\text { - Estudos de análise de riscos e estudos de impactos ambientais; } \\
\text { - Cadastros técnicos multifinalitários; } \\
\text { - Planejamento e Monitoramento de áreas agrícolas. }\end{array}$} \\
\hline
\end{tabular}

Fonte: ImageSat, 2007; Engesat, 2007; Embrapa, 2007. Elaboração e compilação: Fonseca; Matias, 2007.

As imagens deste sensor também podem ser obtidas com correção radiométrica e geométrica. Westin e Forsgren (2002), por exemplo, descrevem um método possível para realizar a ortorretificação das imagens EROS A com acurácia inferior a um pixel; outros estudos relacionados à ortorretificação das imagens EROS foram desenvolvidos em Taiwan (CHEN e TEO, 2004), país que possui inclusive uma antena de recepção das imagens EROS. É também em Taiwan, na National Central University, que foi publicado um artigo descrevendo um experimento na transformação das imagens pancromáticas EROS em imagens 
FONSECA, M.F. \& MATIAS, L.F. Potencial das aplicações de imagens ...

coloridas com perdas não significativas em sua resolução, utilizando para isso uma fusão com imagens multiespectrais do satélite francês SPOT (CHANG e CHEN, 2005).

Esse caso ilustra o que alguns autores vêm enfocando quanto às possibilidades de se extrair informações complementares de imagens oriundas de plataformas orbitais através de técnicas de processamento digital de imagens e da automação parcial de determinadas etapas dos mapeamentos temáticos (VERONESE e FERREIRA, 2006). Apontam, dentre outros parâmetros, a necessidade de se trabalhar com subprodutos dos dados originais, com a fusão de dados de diferentes sensores e com a integração entre as diversas temáticas a serem mapeadas (vegetação, geologia, geomorfologia, uso da terra, entre outros).

A necessidade de minimizar o tempo e os recursos envolvidos faz com que diferentes técnicas sejam testadas, para implementar as aplicações do sensoriamento remoto nestes ambientes (FORESTI e HAMBURGER, 1995). A utilização de imagens de sensoriamento remoto em estudos geográficos torna-se cada vez mais relevante em um momento histórico marcado pelo acentuado dinamismo das transformações sócio-espaciais e pela exigência de se compreender e interpretar as diversas escalas dos fenômenos que se materializam no território.

\section{Potencial das aplicações das imagens do satélite EROS}

A Embrapa Monitoramento por Satélite ${ }^{1}$ vem utilizando imagens EROS na atualização da malha urbana e em estudos de uso e cobertura da terra no município de Campinas, o que destaca sua aplicação em projetos que exigem escalas de detalhe mais acentuadas. Informações obtidas junto a esta instituição apontam para negociações para tentar instalar uma antena de recepção das imagens EROS na sede em Campinas, o que poderia assegurar uma

${ }^{1}$ Empresa Brasileira de Pesquisa Agropecuária, Centro Nacional de Pesquisa de Monitoramento por Satélite - Av. Dr. Júlio Soares de Arruda, 803. Parque São Quirino, CEP 13088-300, Campinas/SP. 
FONSECA, M.F. \& MATIAS, L.F. Potencial das aplicações de imagens ...

maior difusão na divulgação dessas imagens, além de uma significativa ampliação em sua utilização.

Dentre os projetos desenvolvidos e/ou em andamento com o uso das imagens EROS pela Embrapa, pode ser citado o plano emergencial de combate às enchentes para o município de Campinas, que visou identificar áreas de risco no contexto das áreas ocupadas predominantemente pela população mais carente. $\mathrm{O}$ trabalho de levantamento de campo foi auxiliado pelas imagens de alta resolução deste sensor. Além deste projeto, também foram utilizadas imagens EROS para o estudo e monitoramento da mata de Santa Genebra, em Campinas; as imagens possibilitaram, dentre outros aspectos, a observação da densidade da vegetação em pontos específicos.

A utilização conjunta de imagens de satélites de alta e média resolução visando um contexto mais amplo também é fato relevante em trabalho realizado pela instituição no Haiti, país da América Central que está recebendo a Força de Paz comandada por tropas brasileiras. Para mapear e monitorar o país, imagens Landsat $(15 \mathrm{~m})$ foram utilizadas para mapeamentos extensos, imagens Quickbird $(0,61 \mathrm{~m})$ foram utilizadas para detalhar a capital Porto Príncipe, enquanto outras cidades, cobertas por imagens EROS $(1,8 \mathrm{~m})$ foram também mapeadas para missões de reconhecimento (EMBRAPA, 2007).

A importância das imagens de satélite e, sobretudo, dos produtos que podem ser gerados a partir de sua análise, reforçam ainda mais o seu papel dentro do contexto da Geografia; tais iniciativas auxiliam na difusão das imagens como forma de representação e instrumento de inferência nas atividades que envolvem o uso do território. Câmara e Medeiros (2001) já chamavam a atenção para o fato das imagens de satélites servirem como base auxiliar na definição das unidades de paisagem em projetos ambientais.

As imagens do satélite EROS também se mostraram eficazes ao serem utilizadas no mapeamento do uso e ocupação da terra no município de Americana, SP, mais especificamente no entorno do 
FONSECA, M.F. \& MATIAS, L.F. Potencial das aplicações de imagens ...

reservatório de Salto Grande, onde as imagens auxiliaram o trabalho de reconhecimento da área, mapeamento das classes de uso e diagnóstico dos principais conflitos existentes (FONSECA, 2007). Na ocasião, utilizou-se o software $\operatorname{ArcGIS}^{\circledR}$, versão 9.1 (ESRI, 2004) para execução do georreferenciamento e transformação da projeção cartográfica da imagem EROS para possibilitar a compatibilidade dos dados com a base cartográfica, em escala 1:10.000, obtida junto ao Instituto Geográfico e Cartográfico do Estado de São Paulo (IGC), de acordo com padrões cartográficos que, segundo Burrough e McDonnell (1998), atendem um formato de intercâmbio de troca de dados recomendado pelas instituições de vários países e também sugerido para mapeamentos deste tipo. A Figura 01 apresenta um fragmento da imagem EROS correspondente a este estudo.

Figura 01: Área no município de Americana/SP, onde imagens EROS auxiliaram no mapeamento do uso da terra

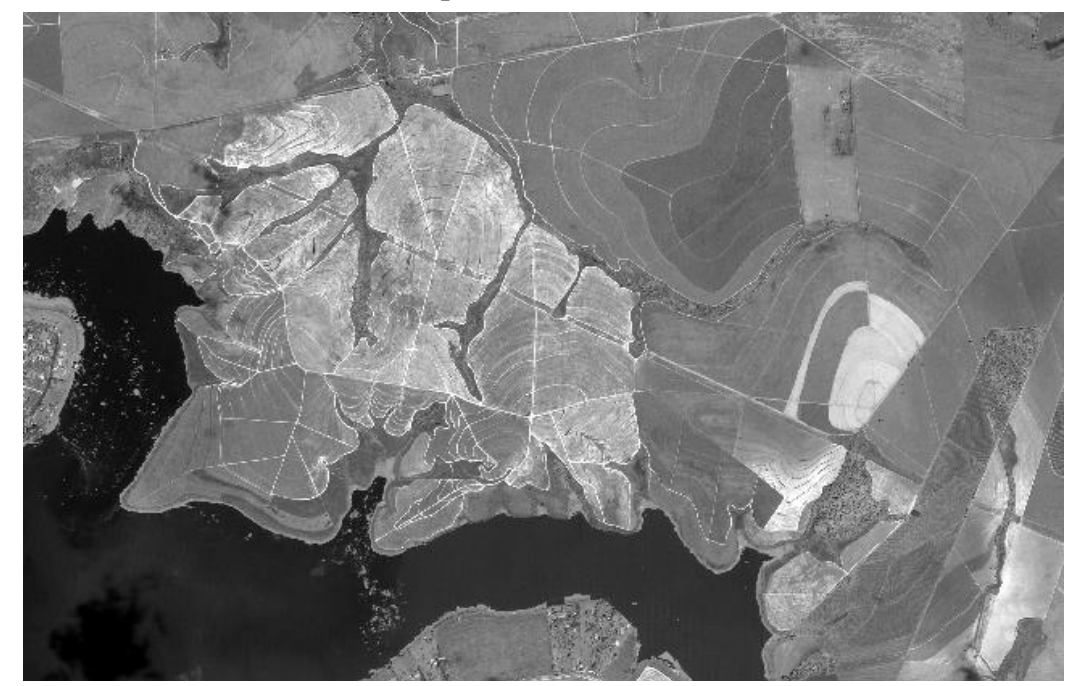

Fonte: Embrapa Monitoramento por Satélite, 2005. 
FONSECA, M.F. \& MATIAS, L.F. Potencial das aplicações de imagens ...

Pelas suas características, as imagens EROS podem ser potencialmente utilizadas como auxílio nas pesquisas em diversas áreas do conhecimento como, por exemplo, para o monitoramento agrícola (levantamento de áreas de plantio, estimativas de áreas cultivadas, identificação de áreas de expansão agrícola) ou em mapeamentos da malha urbana (levantamentos e cadastros técnicos), essencialmente pela sua fina resolução espacial e boa capacidade temporal, o que denota bons indicativos para aplicações com este fim.

$\mathrm{O}$ uso das imagens EROS em diversos países aponta para um leque maior de produtos e subprodutos gerados a partir dessas cenas. São encontrados exemplos na construção de modelos tridimensionais para planejamento urbano, infra-estrutura, desenvolvimento e expansão de redes de utilidades (energia, dutos, tubulações), redes pluviais, plantas industriais, modelos tridimensionais para planejamento de antenas, linhas elétricas, cabos de comunicação, construção e monitoramento de vias férreas, estradas, aeroportos, portos e usinas nucleares (IMAGESAT, 2007).

Alguns recortes de imagens obtidas através deste sensor são apresentados na seqüência, com a aplicação sendo brevemente relatada.

A Figura 02 mostra um recorte de uma cena constituída a partir de imagem EROS referente à cidade de Brasília, capital federal, para fins de identificação de feições urbanas.

A Figura 03 apresenta parte de uma cena da cidade de Ushuaia, no extremo sul da Argentina, situada na costa ocidental da chamada Terra do Fogo. O fragmento da imagem EROS foi utilizada para mapeamento do relevo notadamente íngreme do local e para estudos relacionados à base naval administrada pela marinha argentina (na parte inferior da imagem, observa-se a pista do aeroporto em meio a uma topografia muito particular). 
FONSECA, M.F. \& MATIAS, L.F. Potencial das aplicações de imagens ...

Figura 02: - Brasília (destaque: lago Paranoá e arredores)

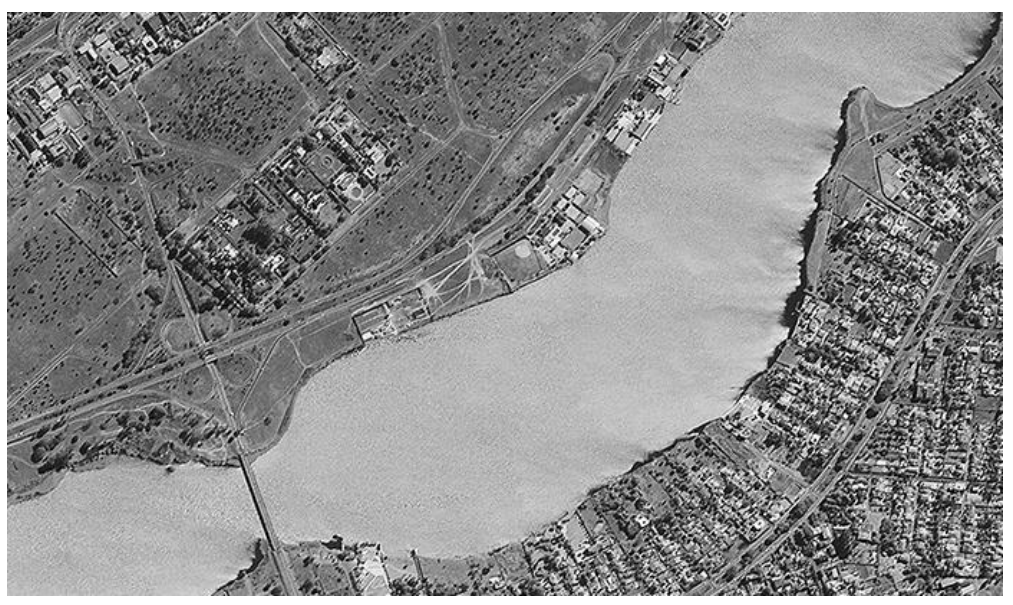

Fonte: Imagesat International, 2007.

Figura 03: Ushuaia, na Argentina, com destaque para pista de pouso local

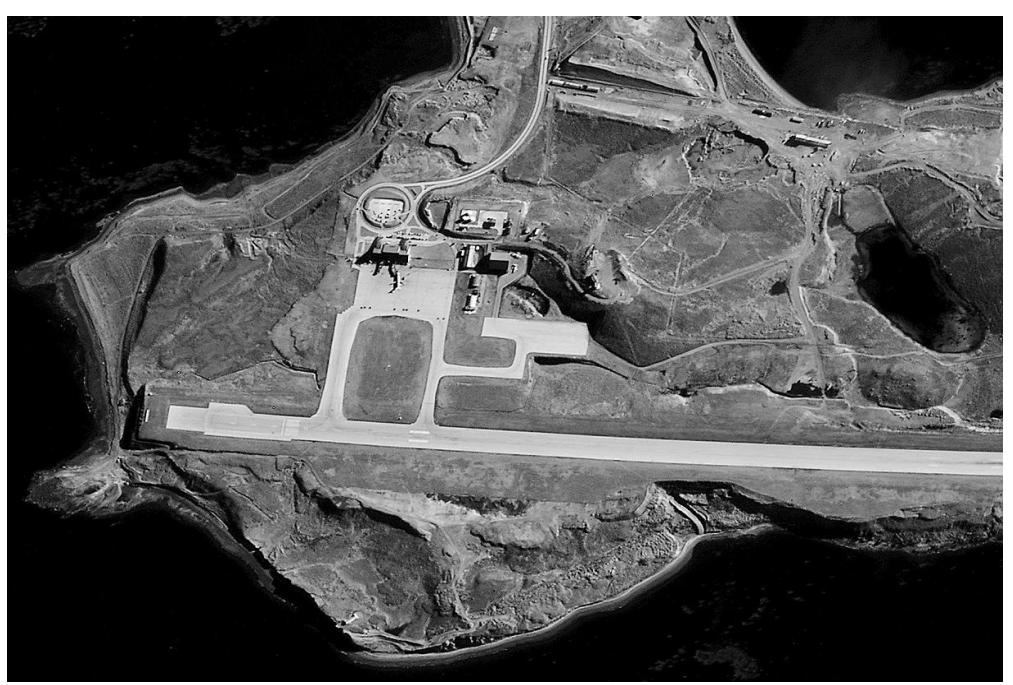

Fonte: Imagesat International, 2007. 
FONSECA, M.F. \& MATIAS, L.F. Potencial das aplicações de imagens ...

A Figura 04 traz uma visão em perspectiva de uma área da cidade de Ploiesti, na Romênia, conhecida atualmente por sua base industrial de destilarias de óleo, engenharia mecânica e fábricas químicas; uma imagem do satélite EROS foi utilizada para estudos de viabilidade ocupacional no entorno do pólo industrial.

Figura 04: Ploiesti, na Romênia: pólo industrial químico

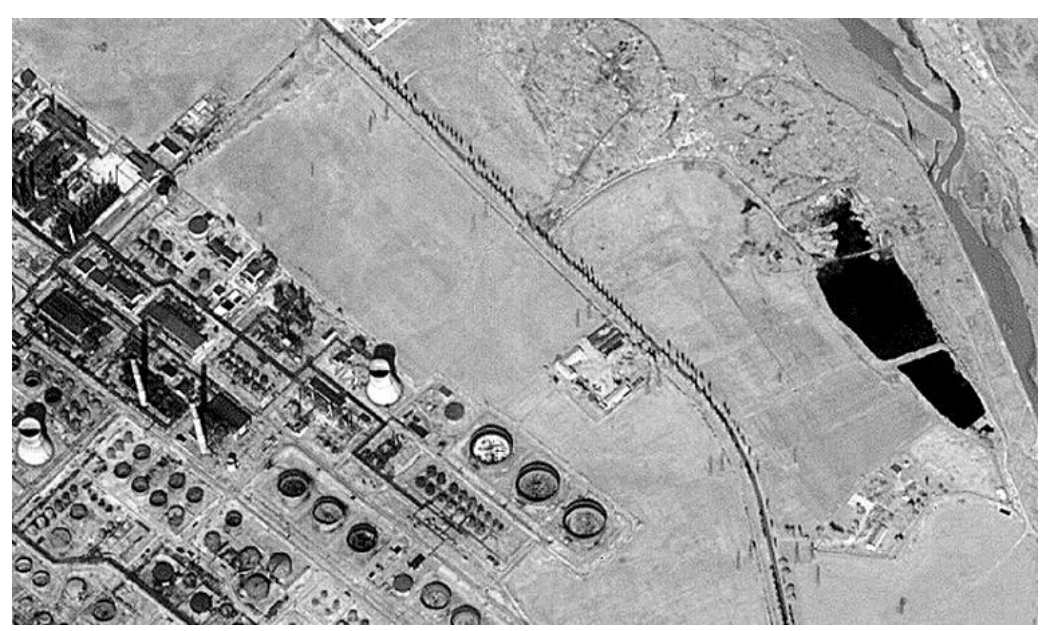

Fonte: Imagesat International, 2007.

A Figura 05 apresenta o uso de imagens EROS em mapeamento para projeto de irrigação. A cena retrata o oásis de Khufrah, na Líbia, onde há produção agrícola em área desértica. 
FONSECA, M.F. \& MATIAS, L.F. Potencial das aplicações de imagens ...

Figura 05: Khufrah, Líbia: irrigação em forma de pivô central

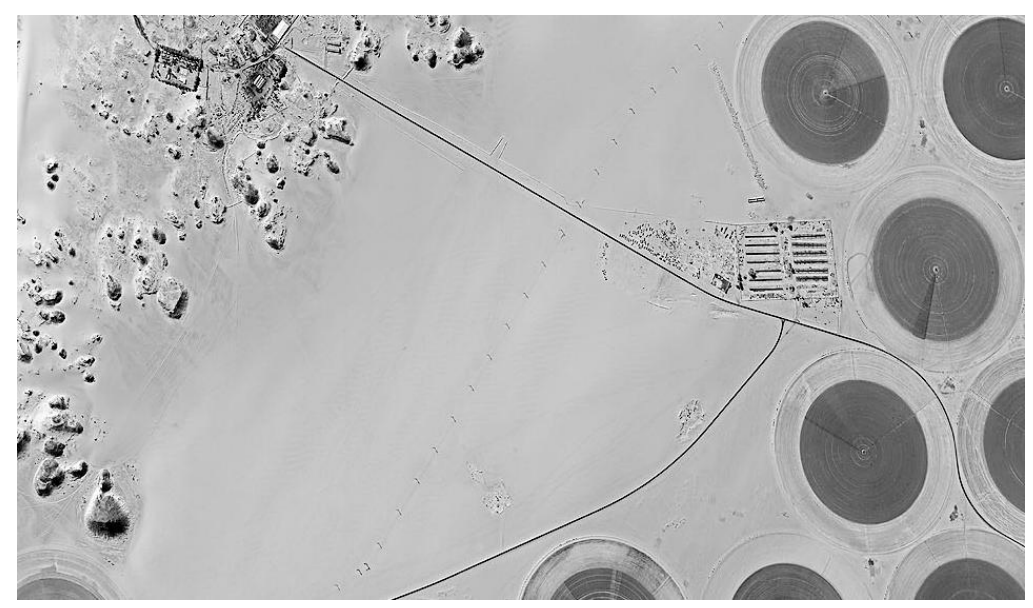

Fonte: Imagesat International, 2007.

\section{Considerações finais}

Além do uso civil, os satélites EROS são parte estratégica da política de inteligência de Israel em monitorar as atividades de desenvolvimento de armas nucleares de países como o Irã, que mantêm relações conturbadas com o governo israelense. Aspecto importante da política nacional de qualquer nação contemporânea, os investimentos em tecnologia espacial colaboram significativamente para o desenvolvimento de novas metodologias para a gestão territorial, como na bem sucedida parceria entre Brasil e China, para ampliação do programa de satélites CBERS (INPE, 2007).

Segundo a ImageSat International, a continuidade do programa EROS está garantida, com a família EROS C planejada para lançamento em 2009, com parâmetros de resolução ainda não definidos.

A crescente demanda por imagens de satélite para estudos geográficos favorece o aparecimento de novos sensores que podem preencher uma lacuna ainda relevante quando se refere a 
FONSECA, M.F. \& MATIAS, L.F. Potencial das aplicações de imagens ...

resoluções espaciais mais refinadas. Além disso, sensores provenientes da tecnologia de radar, ainda de difícil acesso pelos elevados custos, prometem nos próximos anos se tornarem mais uma opção no campo das geotecnologias, já que os mesmos apresentam a capacidade de penetrar nuvens, brumas, fumaça, fenômenos e interferências atmosféricas, e prover uma resolução temporal muito superior (já que não dependem, por exemplo, de fonte solar), além de permitir a extração de características complementares dos alvos (PARADELLA e SANO, 2006).

Ressalta-se, todavia, que a compreensão da realidade sócioespacial vai muito além da discussão técnica de sensores e/ou equipamentos utilizados; ela perpassa, sobretudo, por uma visão mais ampla e pela adoção de uma dialética que permita ao pesquisador de processos geográficos contemplar toda a complexidade e nuances que envolvem uma análise geográfica do território.

É possível que nos próximos anos se tenha cada vez mais disponível uma gama de sensores com resoluções cada vez mais refinadas, e passíveis de serem utilizadas em um intervalo de tempo cada vez mais comprimido. O grande desafio estará em integrar estes dados (ROSA, 1996) e, sobretudo, sistematizar a produção e difusão de conhecimento de modo a tornar suas aplicações cada vez mais contributivas para o bem estar social.

Ao pesquisador sempre caberá, em seu trabalho de investigação, dar um passo adiante, sugerindo e propondo metodologias e interpretações que indiquem um melhor aproveitamento dos dados disponíveis, conduzindo a Geografia ao seu posto de ciência imprescindível na discussão do planejamento e gestão do território. 
FONSECA, M.F. \& MATIAS, L.F. Potencial das aplicações de imagens ...

\section{Referências bibliográficas}

Burrough, P.A., McDonnell, R.A. Principles of geographical information systems - Oxford: Oxford University, 1998.

Câmara, G., Medeiros, J.S. de. Geoprocessamento para projetos ambientais. In Fundamentos de Geoprocessamento, 2001. 36p. Disponível em http://www.dpi.inpe.br.

Chang, L.Y., Chen, C.F., Chen, A.J. An experiment to color EROS panchromatic image. Center for space and remote sensing research, National Central University, Chung Li, Taiwan, 2005.

Chen, L.C., Teo, T.A. Orbit adjustment for EROS A1 high resolution satellite images. Center for space and remote sensing research, National Central University, Chung Li, Taiwan, 2004.

Embrapa Monitoramento por Satélite. Satélites de Monitoramento: EROS. Características dos satélites, principais instrumentos sensores, aplicações. Disponível em http://www.cnpm.embrapa.br. Acessado em 17 de ago. de 2007.

Engesat. EROS - Ficha Técnica Resumida. Disponível em http://www.engesat.com.br. Acessado em 28 de set. de 2007.

ESRI, Environmental Systems Research Institute. Using ArcMap. ESRI: Redlands, 2004.

Fonseca, M.F. Geotecnologias aplicadas ao mapeamento e análise do uso e ocupação da terra no entorno do reservatório de Salto Grande, município de Americana (SP). Relatório parcial de mestrado, Departamento de Geografia do Instituto de Geociências (IG), Universidade Estadual de Campinas (UNICAMP), Campinas, 2007, 36 p.

Foresti, C., Hamburger, D.S. Sensoriamento Remoto aplicado ao estudo do uso do solo urbano, in Tauk-Tornisielo, S.M., Gobbi, N., Fowler, H.G. Análise ambiental: uma visão multidisciplinar. $2^{\circ}$ edição, São Paulo: Editora da Universidade Estadual Paulista, 1995. 
FONSECA, M.F. \& MATIAS, L.F. Potencial das aplicações de imagens ...

Imagesat International. About ImageSat and about Earth Remote Observation Satellite (EROS) - Disponível em http://www.imagesatintl.com. Acessado em 28 de set. de 2007.

Paradella, W.R., Sano, E.E. Aplicações de radar nas geociências e meio ambiente: estado atual e perspectivas, in Chaves, J.M., Rocha, W.J.S da F. Geotecnologias: trilhando novos caminhos nas geociências. Salvador, 2006, 222 p.

Rosa, R., Brito, J.L.S. Introdução ao Geoprocessamento: Sistema de Informação Geográfica. Uberlândia, 1996, 104 p.

Space Imaging International. About Ikonos. Disponível em http://www.spaceimaging.com. Acessado em 28 de set. de 2007.

Veronese, V.F., Ferreira, M.C. Processamento digital de imagens para recursos naturais - uma abordagem integrativa, in Chaves, J.M., Rocha, W.J.S. da F. Geotecnologias: trilhando novos caminhos nas geociências. Salvador, 2006, 222 p.

Westin, T., Forsgren, J. Orthorectification of EROS A1 images. Metria Company, Kiruna, Sweden, 2002.

Recebido em dezembro de 2007 Aceito em outubro de 2008 
